\title{
The first case of Ochrobactrum intermedium bacteremia in a pediatric patient with malignant tumor
}

Wenjing $\mathrm{Wu}^{1,2}$, Yongmei Jiang ${ }^{1,2}$, Wei Zhou ${ }^{1,2}$, Xingxin Liu $\mathrm{u}^{1,2}$ and Linghan Kuang ${ }^{1,2^{*}}$

\begin{abstract}
Background: Ochrobactrum spp. are non-fermenting, Gram-negative bacilli that are regarded as emerging human pathogens of low virulence that can cause infections. The first identified case of Ochrobactrum intermedium was reported in 1998 in a liver transplantation patient with liver abcess. There are no reports of infections in pediatric patients. Here, we report the first case of $O$. intermedium bacteremia in a pediatric patient.

Case presentation: A two and a half years old male was admitted with fever, chills and nausea. He had been diagnosed as pineoblastoma and underwent surgical resection and chemotherapy. O. intermedium was isolated from his blood cultures and identified by matrix-assisted laser desorption/ionization time-of-flight mass spectrometry (MALDITOF MS), however, the Vitek II automated system failed to identify the organism. Then the pathogen was confirmed by $16 \mathrm{~S}$ rDNA sequencing and average nucleotide identity result (ANI) confirmed the precise identification of O. intermedium at genomic level. In addition, the patient recovered well after antibiotic combined therapy.

Conclusions: This, to our knowledge, is the first case of $O$. intermedium bacteremia in a pediatric patient with malignant tumor. Traditional biochemical identification methods such as API 20NE or VITEK2 system cannot differentiate $O$. anthropi and $\mathrm{O}$. intermedium. MALDI-TOF may be a promising tool for rapid identification of microorganisms such as $\mathrm{O}$. intermedium.
\end{abstract}

Keywords: Ochrobactrum, Ochrobactrum intermedium, Bacteremia, Pediatric patient

\section{Background}

Ochrobactrum spp. belongs to the family Brucellaceae and it is widely distributed in the environment and clinical unit, such as soil, plant, water and indwelling catheters [1]. To date, there are 18 Ochrobactrum species with validly published names but only five of them, $O$. anthropi, O. intermedium, O. haematophilum, O. pseudogrignonense and O. pseudintermedium, were isolated from clinical specimens [2]. O. anthropi is the most common species recovered from clinical samples, and

*Correspondence: hanhan840106@163.com

${ }^{1}$ Department of Laboratory Medicine, West China Second University

Hospital, Sichuan University, Chengdu, China

Full list of author information is available at the end of the article a variety of infections, such as catheter-related bloodstream infection, peritonitis or endophthalmitis, could be caused by this pathogen [3]. Unlike O. anthropi, O. intermedium infections were rare and up to now, only nine cases of $O$. intermedium infection were reported, and they usually cause infections in adult patients with bacteremia, prostatic abscess, endophthalmitis and infective endocarditis [2]. Here, we report the first case of O. intermedium bacteremia in a pediatric patient with malignant tumor.

\section{Case presentation}

A two and a half years old male with fever was admitted to our hospital on August 2019. He had been diagnosed as pineoblastoma 7 months ago and underwent surgical 
resection and chemotherapy. He received a ventriculoperitoneal shunt for obstructive hydrocephalus 4 months ago. He presented with fever (up to $40.4{ }^{\circ} \mathrm{C}$ ), chills and nausea 2 days before hospital admission. Physical examination did not reveal any significant findings except pharyngeal hyperemia and swollen tonsils. His breath and heart sounds were normal. No redness or swelling occurred at the peripherally inserted central catheter (PICC) site, which had been in indwelled for 6 months, and the catheter was flushed. Computed tomography, urine examination and cerebrospinal fluid were normal. His white blood cell count and C-reaction protein concentration were $17.6 \times 10^{9} / \mathrm{L}(96.3 \%$ neutrophils $)$ and $130.5 \mathrm{mg} / \mathrm{L}$ (Fig. 1). Three sets of blood cultures were collected from peripheral venipuncture at the beginning of the febrile period, then he was treated with cefoperazone/ sulbactam. Two of the blood cultures were positive and the pathogens were identified as Stenotrophomonas maltophilia by MALDI-TOF MS (bioMérieux, Marcy-l'Étoile, France). Susceptibility testing showed the organism was susceptible to sulfamethoxazole-trimethoprim, minocycline, ceftazidime, levofloxacin, chloramphenicol, and ticarcillin/clavulanate. The antibiotic treatment was changed to the combination of cefoperazone/sulbactam and sulfamethoxazole-trimethoprim. Then the boy remitted and the body temperature dropped to normal range within 3 days since the treatment was adjusted. The white blood cell counts also returned to normal level and repeated blood cultures (collected from peripheral vein) were negative. However, on the 13th day, his temperature increased again with the highest point of $39.1{ }^{\circ} \mathrm{C}$, and blood specimens were collected from two peripheral

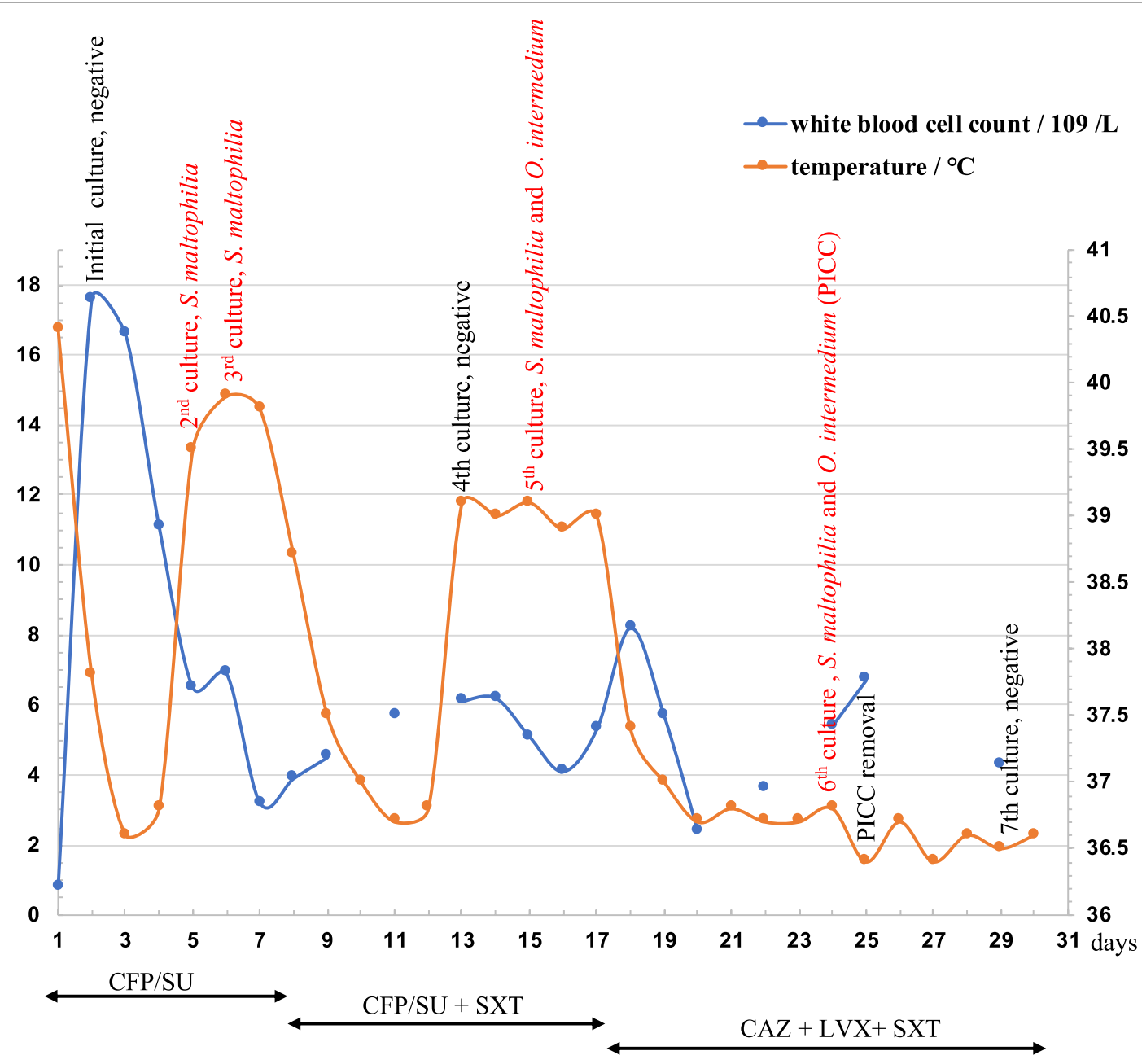

CFP/SU, cefoperazone/sulbactam; SXT, trimethoprim-sulfamethoxazole; LVX, Levofloxacin; CAZ, Ceftazidime.

Fig. 1 Temperature, white blood cell count and the therapeutic schedule of the patient 
veinpuncture sites (left hand and right foot). After $12 \mathrm{~h}$ of incubation, gram-negative bacilli were detected from one blood culture, which was collected from right foot. Two types of colonies grew in the blood agar after cultivation, which were identified as S. maltophilia and O. intermedium (strain 045999) by MALDI-TOF MS with $99.9 \%$ confidence; however, the Vitek II automated system (bioMérieux) failed to identify strain 045999. Genomic DNA of strain 045999 was prepared using the QIAamp DNA mini kit (Qiagen; Hilden, Germany) and were subjected to whole genome sequencing using the HiSeq X10 Sequencer (Illumina; San Diego, CA, USA). For the whole genome sequence of strain $045999,5,619,077$ reads and 1.69 GB bases were generated (coverage, $\times 400$ ), which were assembled into a $4.9 \mathrm{Mb}$ draft genome contains 82 contigs $\geq 200 \mathrm{bp}$ in length (N50, 454,256 bp) with a $57.71 \mathrm{~mol} \%$ GC content. ANI between strain 045999 and the type strain LMG $3301^{\mathrm{T}}$ of O. intermedium (ACQA00000000) was determined using the JSpecies [4]. The ANI value was $97.57 \%$, which was above the cutoff ( $\geq 95-96 \%$ ANI) defining the boundaries of bacterial species [5], confirming that the identification was precise. The same organisms were isolated from the other blood culture collected from left hand after 4 days incubation. Antimicrobial susceptibility testing was performed using broth microdilution with the Vitek II automated system (bioMérieux) and interpreted according to the guidelines for other non-Enterobacterales in CLSI M100 (2019). The strain 045999 was susceptible to sulfamethoxazole-trimethoprim, minocycline, tigecycline, cefepime, levofloxacin, meropenem, imipenem (Table 1). The strain 045999 formed beige, translucent, shiny and mucoid colonies on blood agar within $24 \mathrm{~h}$ of incubation at $35{ }^{\circ} \mathrm{C}$ and gram-staining indicated gram-negative, rod-shaped bacteria without spores. The antimicrobial susceptibility of the $S$. maltophilia was consistent with previous results. According to the results of susceptibility test, the $S$. maltophilia was susceptible to levofloxacin and ceftazidime, and strain 045999 was also susceptible to sulfamethoxazole-trimethoprim and levofloxacin. Therefore, cefoperazone/sulbactam was replaced with levofloxacin and ceftazidime, and sulfamethoxazole-trimethoprim was continued as before. One week after the treatment, blood cultures were obtained from both peripheral venipuncture and PICC. The blood culture obtained from PICC was positive with the growth of S. maltophilia and $O$. intermedium whereas the blood obtained from peripheral venipuncture was negative. Meanwhile, the PICC was removed and the culture was negative. Therefore, the therapy mentioned above was continued for 10 days, and the patient discharged after recovery.

\section{Discussion and conclusion}

Ochrobactrum spp. non-fermenting, Gram-negative bacilli are regarded as emerging human pathogens of low virulence that can cause infections not only in immunocompromised patients but also in human without underlying diseases [6]. Unlike O. anthropi, infection caused by $O$. intermedium is relatively rare. The first identified case of O. intermedium was reported in 1998 in a postliver transplantation patient with a liver abcess, however, the microorganism was wrongly identified as $O$. anthropi by biochemical testing, and it was confirmed to be $O$. intermedium by PCR amplification of $16 \mathrm{~S}$ rDNA gene [7]. But up to now, there were few reports about the $O$. intermedium infection. Based on our literature search in PubMed, only nine cases of O. intermedium infection were reported (Table 2). In the reported cases, O. intermedium causes various infections in immunocompromised patients, such as liver abscess, prostatic abscess, and infective endocarditis [7-9]. Moreover, there were two cases reporting that $O$. intermedium were responsible for causing endophthalmitis and pelvic abscess in immunocompetent hosts $[10,11]$. In two cases, $O$. intermedium was isolated from fecal in patients suffering from either bacteremia or bacteremia associated

Table 1 Antimicrobial susceptibility of the strain 045999

\begin{tabular}{|c|c|c|c|c|c|}
\hline Antimicrobial & $\mathrm{MIC}$ (mg/L) & Category & Antimicrobial & $\mathrm{MIC}(\mathrm{mg} / \mathrm{L})$ & Category \\
\hline Amikacin & 64 & $\mathrm{R}$ & Imipenem & 1 & S \\
\hline Aztreonam & $\geq 64$ & $\mathrm{R}$ & Levofloxacin & 0.25 & S \\
\hline Cefepime & 4 & S & Meropenem & $\leq 0.25$ & S \\
\hline Cefoperazone/sulbactam ${ }^{\mathrm{a}}$ & 32 & । & Minocycline & $\leq 1$ & $\mathrm{~S}$ \\
\hline Ceftazidime & 32 & $\mathrm{R}$ & Piperacillin/tazobactam & $\geq 128$ & $\mathrm{R}$ \\
\hline Ciprofloxacin & $\leq 0.25$ & S & Tigecycline & 0.5 & S \\
\hline Colistin & 64 & $\mathrm{R}$ & Tobramycin & $\geq 16$ & $\mathrm{R}$ \\
\hline Gentamicin & 8 & । & Sulfamethoxazole-trimethoprim & $\leq 1 / 19$ & $\mathrm{~S}$ \\
\hline
\end{tabular}

$S$ susceptible, / intermediate, $R$ resistant

${ }^{a}$ The breakpoint of cefoperazone instead of cefoperazone/sulbactam 
Table 2 Case reports of $O$. intermedium infection

\begin{tabular}{|c|c|c|c|c|c|c|}
\hline Author/Year & Age (year)/sex & Diagnose & Underlying disease & Antibiotic regimen & Prognosis & References \\
\hline Moller et al./1999 & $45 / F$ & Liver abcess & Liver transplant & CIP/metronidazole & Improved & {$[7]$} \\
\hline Apisarnthanarak et al./2005 & $74 / \mathrm{M}$ & Bacteraemia & Bladder cancer & IPM/CIP & Improved & {$[12]$} \\
\hline Vaidya et al./2006 & $49 / \mathrm{M}$ & Pelvic abscess & Non & LVX/metronidazole & Improved & [11] \\
\hline Dharne et al./2008 & $26 / M$ & Non-ulcer dispepsia & Unknown & Unknown & Improved & [19] \\
\hline Jacobs et al./2013 & $34 / \mathrm{M}$ & Endophthalmitis & $\begin{array}{l}\text { Vitrectomy, lensectomy, } \\
\text { removal of intraocular foreign } \\
\text { body }\end{array}$ & Intravitreal MOX & Improved & {$[10]$} \\
\hline $\begin{array}{l}\text { Rodriguez-Villodres } \\
\text { et al./2016 }\end{array}$ & 70/M & Prostatic abscess & Bladder cancer & CIP/cotrimoxazole & Improved & {$[8]$} \\
\hline Bharucha et al./2016 & 23/M & Infective endocarditis & Renal failure & MEM/MIN & Improved & [9] \\
\hline Hirai et al./2016 & $86 / \mathrm{M}$ & Bacteraemia & Cerebral infarction & MEM & Improved & {$[17]$} \\
\hline Kassab et al./2021 & $84 / F$ & Bacteraemia & Primary sclerosing cholangitis & MIN & Improved & {$[13]$} \\
\hline Present case & $2 / \mathrm{M}$ & Bacteraemia & Pineoblastoma & LVX/CAZ/SXT & Improved & - \\
\hline
\end{tabular}

CIP ciprofloxacin, IPM imipenem, LVX levofloxacin, MOX moxifloxacin, MEM meropenem, MIN minocycline, CAZ ceftazidime, SXT sulfamethoxazole-trimethoprim

with liver abscess, indicating that the gastrointestinal tract was possibly the source of infection $[7,12]$. Of the nine cases, seven patients male (so do our case), five patients had bacteremia, two of whom were associated with catheters $[2,13]$. O. anthropi infection is usually seen in immunocompromised patients with indwelling catheters, and catheter-related bacteremia is also most reported in immunocompromised children, especially among boys under 10 years old with malignancies $[6$, 14]. In the present case, similar to the O. anthropi bacteremia, O. intermedium bacteremia occurred in pediatric patient with PICC, who underwent chemotherapy and was immunocompromised. Compared to O. anthropi, O. intermedium infection is rare, and it is likely that some infections thought to be caused by $O$. anthropi may be actually caused by $O$. intermedium, since these species cannot be differentiated by traditional biochemical identification methods such as API 20NE or VITEK2 system leading to misidentification. Thus, the incidence of $O$. intermedium infection in human may be underestimated $[6,15]$. In addition, O. intermedium may undergo an ecological niche specialization and some lineages may shift toward an adaption to the human host based on a phylogenomic approach [16]. In order to accurately identify $O$. anthropi and $O$. intermedium, molecular typing was recommended, however, it was more time-consuming and complicated compared with MALDI-TOF. In this case, the pathogen was initially identified as O. intermedium by MALDI-TOF, which was consistent with previous study $[8,13,17]$. ANI result confirmed the precise identification of $O$. intermedium at genomic level, indicating MALDI-TOF is a promising tool for rapid identification of $O$. intermedium in clinical situations, though more studies are required.
Ochrobactrum isolates were highly resistant to $\beta$-lactams, due to expression of AmpC $\beta$-lactamase, but they were mostly susceptible to levofloxacin, imipenem, ciprofloxacin, TMP-SMX and meropenem [6]. Susceptibility to colistin seems to be a potential marker for distinguishing between $O$. anthropi and $O$. intermedium, as $O$. anthropi is usually sensitive, whereas $O$. intermedium is usually resistant to this class of drugs [6]. In present case, S. maltophilia was isolated along with O. intermedium, indicating that it can cause polymicrobial infections in bloodstream infection (BSI), but O. anthropi seldom cause polymicrobial infections with other bacteria in BSI. Thus, the difficulty of treatment was increased. Catheter-related BSI was highly suspected in this case, but it was denied, because the blood culture obtained from PICC was positive, however, blood culture obtained from peripheral venipuncture and catheter culture were both negative. This is likely due to the use of antibiotics, therefore, the correct collection location, timing or times of blood sample play important roles in diagnosis of BSI infections [18]. Although both the two isolates were susceptible to sulfamethoxazole-trimethoprim, the combination treatment (levofloxacin, ceftazidime and sulfamethoxazole-trimethoprim) was used in our case and showed a good response.

In conclusion, here we reported the first case of $O$. intermedium bacteremia in a pediatric patient with malignant tumor. Infection caused by $O$. intermedium is uncommon, however, the incidence is probably being underestimated due to the difficult identification, and the clinical significance of $O$. intermedium remains unclear. Currently, the identification of O. intermedium is confirmed by molecular biological technique, however, this method may not be available in many clinical 
laboratories. Instead, MALDI-TOF MS could be helpful in identifying $O$. intermedium.

\author{
Abbreviations \\ MALDI-TOF MS: Matrix-assisted laser desorption/ionization time-of-flight \\ mass spectrometry; ANI: Average nucleotide identity result; CLSI: Clinical \\ Laboratory Standards Institute; PICC: Peripherally inserted central catheter; BSI: \\ Bloodstream infection.
}

\section{Acknowledgements}

Not applicable.

\section{Authors' contributions}

WJW and LHK designed the study, analyzed the data, and wrote the manuscript. WZ, XXL and YMJ analyzed the data and reviewed the manuscript. All authors read and approved the final manuscript.

\section{Funding}

This work was supported by the Key R\&D project of Science \& technology Department of Sichuan Province (Grant no. 2020YFS0081), Science and Technology Department of Sichuan Province (Grant no. 2020YFS0100), Science and Technology Bureau of Chengdu (Grant no. 2019-YF05-01178-SN), National Natural Science Foundation of China (Grant no. 32070747) and the Popularization and Application project of Health Commission of Sichuan Province (Grant no. 19PJ070). The funders support partial testing item fee and have no role in the design of the study, analysis, and in writing the manuscript.

\section{Availability of data and materials}

The draft genome sequence of strain 045999 has been deposited into GenBank/DDBJ/EMBL under Accession number JABXOG000000000.

\section{Declarations}

Ethics approval and consent to participate

Not applicable.

\section{Consent for publication}

Written informed consent was obtained from the child's parent for publication of this report and any accompanying images.

\section{Competing interests}

The authors declare no conflicts of interests.

\section{Author details}

${ }^{1}$ Department of Laboratory Medicine, West China Second University Hospital, Sichuan University, Chengdu, China. ${ }^{2}$ Key Laboratory of Birth Defects and Related Diseases of Women and Children (Sichuan University), Ministry of Education, Chengdu, China.

Received: 12 December 2020 Accepted: 2 December 2021

Published online: 14 December 2021

\section{References}

1. Mahmood MS, Sarwari AR, Khan MA, Sophie Z, Khan E, Sami S. Infective endocarditis and septic embolization with Ochrobactrum anthropi: case report and review of literature. J Infect. 2000;40(3):287-90.

2. Ryan MP, Pembroke JT. The genus Ochrobactrum as major opportunistic pathogens. Microorganisms. 2020;8(11):1797.

3. Alparslan C, Yavascan O, Kose E, Sanlioglu P, Aksu N. An opportunistic pathogen in a peritoneal dialysis patient: Ochrobactrum anthropi. Indian J Pediatr. 2013;80(1):72-4.

4. Richter M, Rossello-Mora R, Oliver Glockner F, Peplies J. JSpeciesWS: a web server for prokaryotic species circumscription based on pairwise genome comparison. Bioinformatics. 2016;32(6):929-31.
5. Richter M, Rossello-Mora R. Shifting the genomic gold standard for the prokaryotic species definition. Proc Natl Acad Sci USA. 2009:106(45):19126-31.

6. Thoma B, Straube E, Scholz HC, Al Dahouk S, Zoller L, Pfeffer M, Neubauer $\mathrm{H}$, Tomaso H. Identification and antimicrobial susceptibilities of Ochrobactrum spp. Int J Med Microbiol. 2009;299(3):209-20.

7. Moller LV, Arends J, Harmsen H, Talens A, Terpstra P, Slooff M. Ochrobactrum intermedium infection after liver transplantation. J Clin Microbiol. 1999:37(1):241-4.

8. Rodriguez-Villodres A, Cuevas Palomino A, Gomez Gomez MJ. Usefulness of MALDI-TOF mass spectrometry in infections by infrequent microorganisms such as Ochrobactrum intermedium. Med Clin (Barc). 2016;147(6):277-8.

9. Bharucha T, Sharma D, Sharma H, Kandil H, Collier S. Ochromobactrum intermedium: an emerging opportunistic pathogen-case of recurrent bacteraemia associated with infective endocarditis in a haemodialysis patient. New Microbes New Infect. 2017;15:14-5

10. Jacobs DJ, Grube TJ, Flynn HW Jr., Greven CM, Pathengay A, Miller D, Sanke RF, Thorman J. Intravitreal moxifloxacin in the management of Ochrobactrum intermedium endophthalmitis due to metallic intraocular foreign body. Clin Ophthalmol. 2013;7:1727-30.

11. Vaidya SA, Citron DM, Fine MB, Murakami G, Goldstein EJ. Pelvic abscess due to Ochrobactrum intermedium [corrected] in an immunocompetent host: case report and review of the literature. J Clin Microbiol. 2006:44(3):1184-6.

12. Apisarnthanarak A, Kiratisin P, Mundy LM. Evaluation of Ochrobactrum intermedium bacteremia in a patient with bladder cancer. Diagn Microbiol Infect Dis. 2005;53(2):153-5.

13. Kassab I, Sarsam N, Affas S, Ayas M, Baang JH. A case of Ochrobactrum intermedium bacteremia secondary to cholangitis with a literature review. Cureus. 2021:13(4):e14648.

14. Saavedra J, Garrido C, Folgueira D, Torres MJ, Ramos JT. Ochrobactrum anthropi bacteremia associated with a catheter in an immunocompromised child and review of the pediatric literature. Pediatr Infect Dis J. 1999; 18(7):658-60.

15. Teyssier C, Marchandin H, Jean-Pierre H, Diego I, Darbas H, Jeannot JL, Gouby A, Jumas-Bilak E. Molecular and phenotypic features for identification of the opportunistic pathogens Ochrobactrum spp. J Med Microbiol. 2005;54(Pt 10):945-53.

16. Leclercq SO, Cloeckaert A, Zygmunt MS. Taxonomic organization of the family Brucellaceae based on a phylogenomic approach. Front Microbiol. 2019;10:3083.

17. Hirai J, Yamagishi Y, Sakanashi D, Koizumi Y, Suematsu H, Mikamo H. A case of bacteremia caused by Ochrobacterium intermedium. Kansenshogaku Zasshi. 2016;90(2):129-33.

18. Kirn TJ, Weinstein MP. Update on blood cultures: how to obtain, process, report, and interpret. Clin Microbiol Infect. 2013;19(6):513-20.

19. Kulkarni GJ, Shetty S, Dharne MS, Shouche YS. Genome sequencing analysis reveals virulence-related gene content of Ochrobactrum intermedium strain 229E, a urease-positive strain isolated from the human gastric niche. FEMS Microbiol Lett. 2014;359(1):12-5.

\section{Publisher's Note}

Springer Nature remains neutral with regard to jurisdictional claims in published maps and institutional affiliations. 